\title{
NUMERICAL STUDY AND ANALYSIS OF FUNCTIONALLY GRADED DOUBLE CURVED PANELS UNDER FREE VIBRATION
}

\author{
RAPARTHI SRILAKSHMI ${ }^{1} \&$ C. H. RATNAM ${ }^{2}$ \\ ${ }^{1}$ Research Scholar, Department of Mechanical Engineering, Andhra University, Visakhapatnam, India \\ ${ }^{2}$ Professor, Department of Mechanical Engineering, Andhra University, Visakhapatnam, India
}

\begin{abstract}
The primary objective of this study is to investigate the linear and free vibration analysis of functionally graded doublecurved shells, using higher-order shear deformation theory using Matlab numerical programming. The mechanical properties of the plate are supposed to vary continually in the direction of thickness and the volume fractions of the constituents are obtained using two types of four-parameter power-law distribution. A continuous isoparametric lagrangian model is used to develop fundamental equations for FGM shells. Convergence tests carried out, followed by an extensive literature validation to highlight the accuracy and efficiency of the current FG model. Two types of FGM combinations namely $\mathrm{Al} / \mathrm{Al}_{2} \mathrm{O}_{3}, \mathrm{Al} / \mathrm{ZrO}_{2}$, with boundary conditions, geometric parameters, and the consequence of the four parameters are considered for the present study. Finally, some numerical problems were addressed with different volume fractions and boundary conditions to investigate the frequency response of double-curved shells.
\end{abstract}

KEYWORDS: Functionally graded materials, HSDT, Curved shells\&Four parameter power law

Received: May 19, 2020; Accepted: Jun 09, 2020; Published: Jun 24, 2020; Paper Id.: IJMPERDAUG202013

\section{INTRODUCTION}

A group of scientist from Japan first observed the importance of functionally graded materials (FGMs)for a thermal barrier application. FGMs are a blend of two constituents, such as ceramics and metals to resist high-temperature and toughness. FGMs show a smooth and constant difference in material properties through-thickness. FGMs arrangement discards stress concentrations and reducesdelamination effect in layered composites. FGMs are quickly extending for engineering applications, which is fundamental for engineers. Several kinds of research produced different hypotheses for analyzing plates and shells structures.

Pandya and kant[1]developed FE based composite plates under transverse load based on HOSDT.Reddy[2]presented theoretical formulations based on TOSDT and Finite element analysis accounts geometric nonlinearity and thermo coupling and Navier rectangular plates. The author found the gradient composition playsan important role in estimatingthe performance of the plate under shear. Reddy and cheng[3]presented vibration analysis of FGM shallow shell as a polygonal form quiescent on WinklerPasternak elastic foundation and the formulation based on CPT,FSDTand HSDT and Mori Tanaka observed frequencies are extracted different flexural,stretching,thickness shear mode conditions.Batra and Aimmanee[4]analysed free vibration behaviour and stress distribution in a thick isotropic and homogeneous plate. Kim [5] developed temperature dependent FG plates vibration analysis using Rayleigh-Ritz procedure. Temperature and heat flow thermal conditions observed. Kandasamy and Singh [6] investigated the vibration of cylindrical deep shells with skewed open circular. Tornabene and Viola[7]investigated the dynamic behaviour of parabolic shell structures using 
Generalized Differential Quadrature.

Zhao et al. [8] presented free FG plate vibration analysis based on FOSDT is used to address the rotary inertia,transverse shear strain and frequency characteristics.Tornabene[9] investigated double curved shells using GDQ andFOSDT. Hosseini- Hashemi et al [10]worked onmoderately thick plates to observe free vibration analysis.Talha and Singh [11]studied free vibration and static analysis of FG plates using HOSDT conjunction with FE.The material properties of the plate presumed to differ continuously in the direction of thickness by simple application of the power law and various parameters are observed. Liew et al[12] presented a review ofthe meshless method. Ruan et al[13]studied uniformly distributed FGM skew plate by the Kirchhoff thin plate theory which is subjected to tangential follower force are derived.Tornabene et al. [14] studied using the Generalized Differential Quadrature Approach used to test rectangular and annular composite laminates shells.Jha et al.[15]presented a brief review ofthe FG application and various studies on FGM plates.

Shariyat and Hosseini[16] investigated composite sandwich plates with viscoelastic core under biaxial loads. GulshanTaj[17]presented the free vibration ofFGM skew plates under the thermal environment condition and based on the HOSDT.Xie et al[18]presented free vibration on FGM parabolic and spherical shells using haar wavelet discretization and FSDT of revolution with classical and elastic boundary conditions. Kar and Panda[19]investigated FG curved panel free vibration response of shear deformable under the linear, nonlinear, uniform, temperature fields at different geometric parameters are observed. Zhang et al[20]presented circular cylindrical double-shells under free and forced vibration . Wang et al[21]investigated on vibration behavior of different double curved shellsbased on first order deformation theory, and four parameter power law distribution.

Tornabene et al[22]studied FGMs of doubly-curved shells for vibrational behavior including porosity and formulation based on the FOSDT and Navier solution method.Rezaei et al[23]presented based on FOSDT for rectangular plates under free vibration analysis of FG materials with porosities. Moita et al[24]developed vibration response with active and passive damping multi-player sandwich plate/shell embedded with FGM-Piezoelectric viscoelastic core, with plates using different boundary conditions. The material model formulation is based on shear deformation theories.Chakravarty and Pradhan[25] investigated FG thin plates lay on Winkler and Pasternak elastic foundation. Based on CPT and RayleighRitz method,Mouli and Koona[26]investigated on FG curved panels for vibration characteristics of rectangular and skew forms. Governing equations comprehended by Hamilton's standard and TSDT.Katariya and Panda [27][28] examined skew sandwich flat and curved panel for static analysis to evaluate the response of sandwich panel proposed mathematically higher-order kinematics midplane theory and Green-Lagrange strain displacement relation for the different configuration. Rezaiee-Pajand[29]studied FG shells under different loading conditions based on FEM Voigt's model on thermo-elastic nonlinear analysis.From the literature, extensive study available on mathematical model developments of FGM shells and analytical models with different shear deformation theories. However, very few works are observed on free vibration analysis of FGM shells. Hence in the present study, a linear vibration analysis was carried out for different mode shapes with different power law index were considered to estimate the response. 


\section{GEOMETRIC CONFIGURATION AND MATERIAL PROPERTIES}
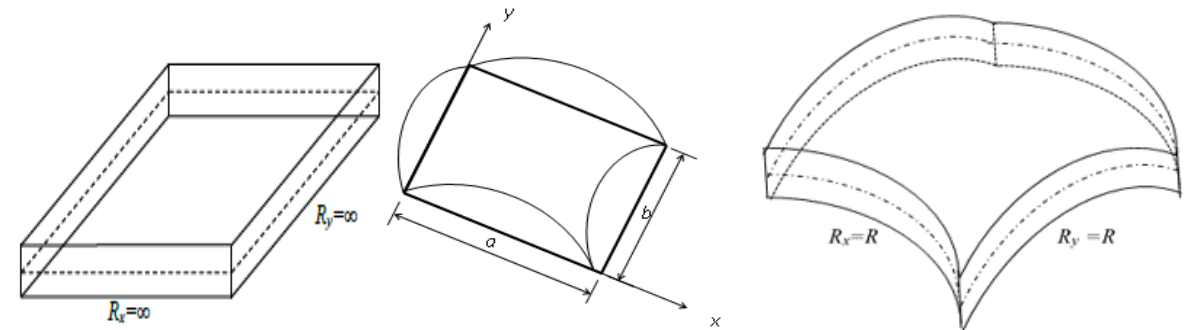

Flat (a)Dimensions of a Flat Panel (b)Spherical (c)
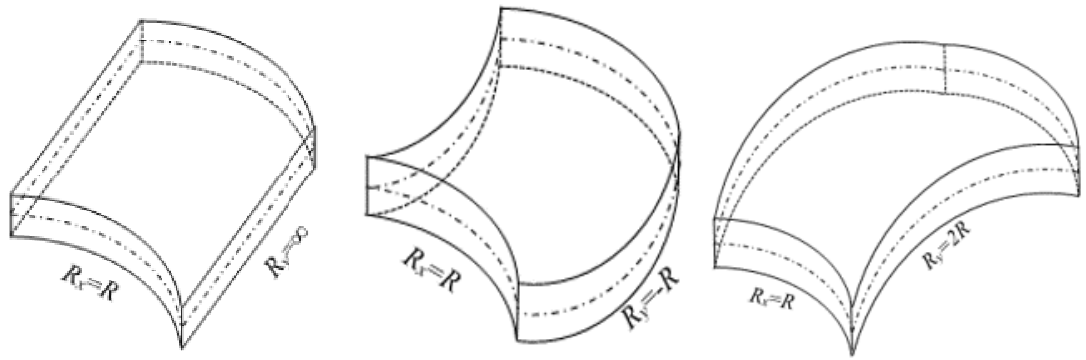

Cylindrical (d)Hyperboloid (e)Elliptical (f)

Figure 1: Shapesof various Shell Configurations Generated from a General Doubly-curved shell [19]
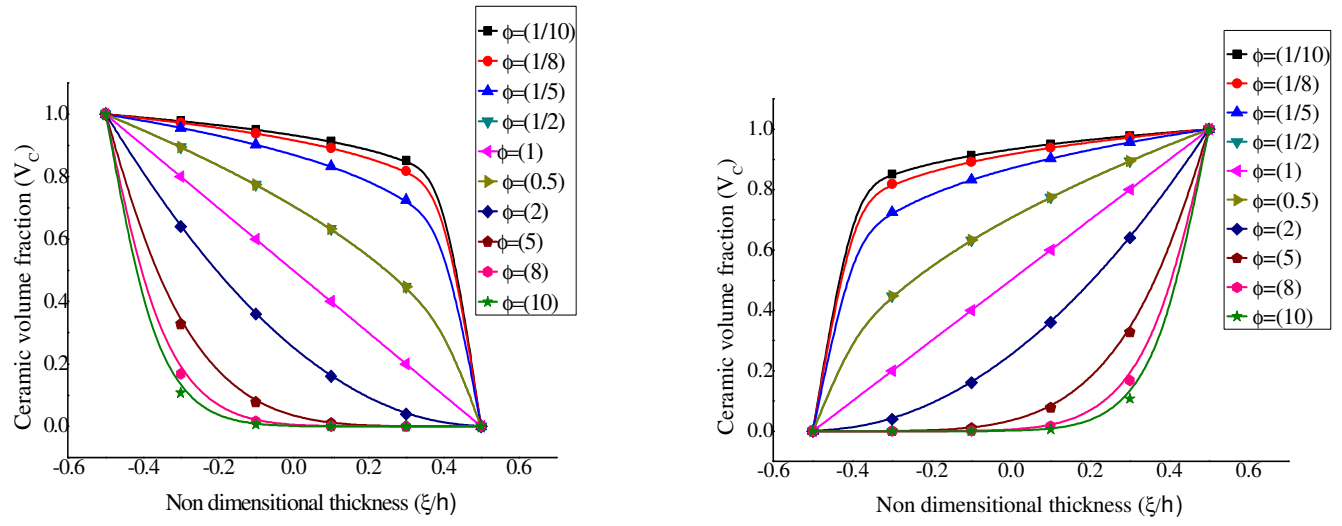

(a) (b)

Figure 2: Variation of Volume Fraction through Thickness Direction for various values of Power Law Index.

Usually, functionally graded materials consist of a two-component mixture. In this work, the functionally graded material is presumed to be made of a combination of metal and ceramic components.

Table 1: Metal and Ceramics Material Properties.[8]

\begin{tabular}{|l|l|c|c|c|}
\hline \multirow{2}{*}{ Composition } & Materials & $\begin{array}{c}\text { Young's Modulus } \\
(\mathbf{G P a})\end{array}$ & Mass Density m(kg/m3) & Poisson's Ratio \\
\hline Metal & Aluminium $(\mathrm{Al})$ & 70 & 2702 & 0.3 \\
\hline \multirow{2}{*}{ Ceramic } & Zirconia $\left(\mathrm{ZrO}_{2}\right)$ & 151 & 3000 & 0.3 \\
\cline { 2 - 5 } & Alumina $\left(\mathrm{Al}_{2} \mathrm{O}_{3}\right)$ & 380 & 3800 & 0.3 \\
\hline
\end{tabular}

In FG shells, the physical properties vary constantly and smoothly from thickness direction $(\xi)$ to the function of volume fractions of its components. The Young module $\mathrm{E}$ and mass density $\rho$ Poisson ratio $\mu$ can be conveyed as a linear function. 


$$
\begin{gathered}
\mathrm{E}(\xi)=\left(E_{c}-E_{m}\right) \mathrm{V}_{c}+\mathrm{E}_{m} \\
\rho(\xi)=\left(\rho_{c}-\rho_{m}\right) \mathrm{V}_{c}+\rho_{m} \\
\mu(\xi)=\left(\mu_{c}-\mu_{m}\right) \mathrm{V}_{c}+\mu_{m}(1 c)
\end{gathered}
$$

First, two separate power-law differences in the thickness-direction of the volume fraction of the constituents are considered. The influence on the mechanical behaviour of functionally graded shells is explored by the power-law exponent and one of the power-law distribution choices.

The base surface of the structure is ceramic rich for the first power-law application, while the top surface can be metal rich as in Fig.2 (a) shown The second power law distribution 2(b) indicates that, for the subsequent distribution, the top surface of the plate $(\xi / \mathrm{h}=0.5)$ is ceramic rich, while the base surface of the plate $(\xi / \mathrm{h}=-0.5)$ is rich in metal. [9]

$$
\begin{aligned}
& \operatorname{FGM}_{1(\mathrm{i} / \mathrm{j} / k / \varphi)}: \mathrm{V}_{c}=\left(1-\mathrm{i}\left(\frac{1}{2}+\frac{\xi}{h}\right)+\mathrm{j}\left(\frac{1}{2}+\frac{\xi}{h}\right)^{\mathrm{k}}\right)^{\varphi} \\
& \mathrm{FGM}_{2(\mathrm{i} / j / k / \varphi)}: \mathrm{V}_{c}=\left(1-\mathrm{i}\left(\frac{1}{2}-\frac{\xi}{h}\right)+\mathrm{j}\left(\frac{1}{2}-\frac{\xi}{h}\right)^{\mathrm{k}}\right)^{\varphi}
\end{aligned}
$$

The parameters $\mathrm{i}, \mathrm{j}, \mathrm{k}$ dictates the material variation profile through the functionally graded shell thickness.

The volume fractions of all the constituent is given by

$$
\mathrm{V}_{c}+\mathrm{V}_{m}=1
$$

\section{THEORETICAL FORMULATIONS}

Figure 1, shows general doubly curved FGP of uniform thickness ' $h$ ' with a rectangular base of sides $a$ and $b . R_{x}$ and $R_{y}$ are principal radii of curvature of the shell along $x$ and $y$ directions, respectively. The respective radii of curvature of flat,spherical, cylindrical,hyperbolic paraboloid and ellipsoid shells are expressed as $R_{x}=R_{y}=\infty ; R_{x}=R_{y}=R ; R_{x}=R, R_{y}=\infty ; R_{x}=R$, $R_{y}=-R$; and $R_{x}=R, R_{y}=2 R$ is shown in Figure1(a),(c),(d), (e) and (f).

The displacement of FG curved Shell is obtained based onHSDT kinematics in the mid-plane of the Shell (at $z=$ $0)$. Global displacements $(u, v, w)$ are defined in the polynomial form of nine unknown parameters $\left(u_{0}, v_{0}, w_{0}, \theta_{x}, \theta_{y}, u_{0}^{*}, v_{0}^{*}, \theta_{x}^{*}, \theta_{y}^{*}\right)$ as in Pandya and Kant[1]

$$
\left.\begin{array}{l}
\Pi(x, y, z)=\Pi_{0}(x, y)+\xi \theta_{x}(x, y)+\xi^{2} \Pi_{0}^{*}(x, y)+\xi^{3} \theta_{x}^{*}(x, y) \\
\Theta(x, y, z)=\Theta_{0}(x, y)+\xi \theta_{y}(x, y)+\xi^{2} \Theta_{0}^{*}(x, y)+\xi^{3} \theta_{y}^{*}(x, y) \\
\Omega(x, y, z)=\Omega_{0}(x, y)
\end{array}\right\}
$$

where, $\left(\Pi_{0}, \Theta_{0}, \Omega_{0}\right)$ denote the mid-plane displacements along $(x, y, z)$ coordinates, respectively. $\theta_{y}$ and $\theta_{x}$ are the shear rotations about the $x$ - and $y$-axis, respectively,and $\left(\Pi_{0}^{*}, \Theta_{0}^{*}, \theta_{x}^{*}, \theta_{y}^{*}\right)$ are the higher-order terms defined in the mid-plane of the curved panel. These nine unknowns can be defined in the mid-plane, i.e., at $z=0$ as $\Pi_{0}=\Pi(x, y)$, 
$\Theta_{0}=\Theta(x, y), \Omega_{0}=\Omega(x, y), \quad \theta_{x}=\frac{\partial \Pi}{\partial z}, \theta_{y}=\frac{\partial \Theta}{\partial z}, \quad \Pi_{0}^{*}=\frac{1}{2}\left(\frac{\partial^{2} \Pi}{\partial z^{2}}\right), \quad \Theta_{0}^{*}=\frac{1}{2}\left(\frac{\partial^{2} \Theta}{\partial z^{2}}\right), \theta_{x}^{*}=\frac{1}{6}\left(\frac{\partial^{3} \Pi}{\partial z^{3}}\right) \quad$ and $\theta_{y}^{*}=\frac{1}{6}\left(\frac{\partial^{3} \Theta}{\partial z^{3}}\right)$

The linear strains are given by $\left.\left\{\varepsilon_{\}}\right\}=\left\{\begin{array}{l}\varepsilon_{x x} \\ \varepsilon_{y y} \\ \varepsilon_{x y} \\ \varepsilon_{x z} \\ \varepsilon_{y z}\end{array}\right\}=\left\{\begin{array}{l}\left(\frac{\partial \Pi}{\partial x}+\frac{\Omega}{R}\right) \\ \left(\frac{\partial \Theta}{\partial y}+\frac{\partial}{R_{y}}\right) \\ \left(\frac{\partial \Theta}{\partial x}+\frac{2 \Omega}{R_{x y}}\right) \\ \left(\frac{\partial \Pi}{\partial z}+\frac{\partial \Omega}{\partial x}-\frac{\Pi}{R}\right) \\ \left(\frac{\partial \Theta}{\partial z}+\frac{\partial \Omega}{\partial y}-\frac{\Theta}{R}\right)\end{array}\right)\right\}$

Now, replacing the displacements as in Equation (5)into the Equation (6) the terms of the strain may be defined.

$$
\begin{aligned}
& \left\{\begin{array}{l}
\varepsilon_{x x} \\
\varepsilon_{y y} \\
\gamma_{x y} \\
\gamma_{x z} \\
\gamma_{y z}
\end{array}\right\}=\left\{\begin{array}{l}
\varepsilon_{x}{ }^{0} \\
\varepsilon_{y}{ }^{0} \\
\varepsilon_{x y}{ }^{0} \\
\varepsilon_{x z}{ }^{0} \\
\varepsilon_{y z}{ }^{0}
\end{array}\right\}+z\left\{\begin{array}{l}
k_{x}{ }^{1} \\
k_{y}{ }^{1} \\
k_{x y}{ }^{1} \\
k_{x z}{ }^{1} \\
k_{y z}{ }^{1}
\end{array}\right\}+z^{2}\left\{\begin{array}{l}
k_{x}{ }^{2} \\
k_{y}{ }^{2} \\
k_{x y}{ }^{2} \\
k_{x z}{ }^{2} \\
k_{y z}{ }^{2}
\end{array}\right\}+z^{3}\left\{\begin{array}{l}
k_{x}{ }^{3} \\
k_{y}{ }^{3} \\
k_{x y}{ }^{3} \\
k_{x z}{ }^{3} \\
k_{y z}{ }^{3}
\end{array}\right\} \\
& \varepsilon=\varepsilon^{0}+\xi k^{1}+\xi^{2} k^{2}+\xi^{3} k^{3}
\end{aligned}
$$

Where the strain terms of the superscript $0,1,2,3$ are the extension, mid-plane $\varepsilon^{0}, k^{1}, k^{2}$, and $k^{3}$ are the mid-plane strain, curvature, and higher-order terms [19]

The final form of equilibrium equation for the vibrated FGP can be written as below:

$$
\left(\left(\left[K_{g l s}\right]-\left[K_{G M}\right]\right)-\omega_{n}^{2}[M]\right)\{\Lambda\}=0
$$

Where, $\omega_{n}$ is the eigenvalue (natural frequency) of the free vibrated FGP while $\Lambda$ is the corresponding eigenvector (mode shapes).

The free vibration analysis of the non-dimensional frequency parameter $\bar{\omega}$ expressed as below

$$
\left(\bar{\omega}=\omega\left(a^{2} / h\right) \sqrt{\rho_{c} / E_{c}}\right)
$$




\section{RESULTS AND DISCUSSIONS}

\subsection{Study of Convergence and Comparison}

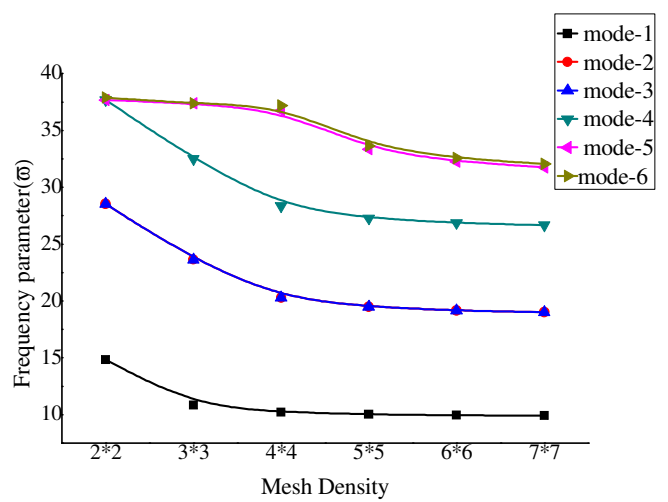

The first step, the number of nodes needed for the convergence analysis of the present model for the determination of the uniform mesh refining computed for the $\mathrm{FG}(\mathrm{Al} / \mathrm{ZrO} 2)$. CCCC condition and $(\varphi=0)((6 * 6)$ mesh size chosen. For accuracy of the model Table, 2,3, and 4 demonstrate a comparison study and good agreement observed from available solutions based on FOSDT.

Table 2: Variation of the Frequency Parameter $\left(\bar{\omega}=\omega\left(a^{2} / h\right) \sqrt{\rho_{c} / E_{c}}\right)$ with the Volume Fraction Exponent $\varphi$ for plate $\mathrm{Al} / \mathrm{Al}_{2} \mathrm{O}_{3}(\mathbf{a} / \mathbf{h}=10)$

\begin{tabular}{|c|c|c|c|c|c|c|c|c|}
\hline \multirow[b]{2}{*}{$\begin{array}{c}\text { FGM } \\
\text { TYPE }\end{array}$} & \multirow[b]{2}{*}{ BCC } & \multirow[b]{2}{*}{ MODE } & \multicolumn{3}{|c|}{0} & \multicolumn{3}{|c|}{0.5} \\
\hline & & & Present & $\begin{array}{c}\text { Zhao } \\
\text { et. al. } \\
{[8]}\end{array}$ & \%ERROR & Present & $\begin{array}{c}\text { Zhao } \\
\text { et. al. } \\
{[8]}\end{array}$ & \%ERROR \\
\hline \multirow{8}{*}{ FGM1 } & \multirow{4}{*}{ SSSS } & 1 & 5.7670 & 5.6763 & 1.5979 & 5.1920 & 5.1105 & 1.5948 \\
\hline & & 2 & 13.8271 & 13.5370 & 2.1430 & 12.4702 & 12.2070 & 2.1561 \\
\hline & & 3 & 13.8283 & 13.5370 & 2.1519 & 12.4713 & 12.2070 & 2.1652 \\
\hline & & 4 & 19.4217 & 20.6330 & -5.8707 & 17.8909 & 18.6300 & -3.9673 \\
\hline & \multirow{4}{*}{$\mathrm{CCCC}$} & 1 & 9.9436 & 9.6354 & 3.1986 & 8.9816 & 8.7011 & 3.2237 \\
\hline & & 2 & 19.1612 & 18.3050 & 4.6774 & 17.3493 & 16.5670 & 4.7220 \\
\hline & & 3 & 19.1612 & 18.3050 & 4.6774 & 17.3493 & 16.5670 & 4.7220 \\
\hline & & 4 & 26.8640 & 25.5060 & 5.3242 & 24.3528 & 23.1110 & 5.3732 \\
\hline \multirow{8}{*}{ FGM2 } & \multirow{4}{*}{ SSSS } & 1 & 5.7670 & 5.6763 & 1.5979 & 5.1926 & 5.1105 & 1.6065 \\
\hline & & 2 & 13.8271 & 13.5370 & 2.1430 & 12.4730 & 12.2070 & 2.1791 \\
\hline & & 3 & 13.8283 & 13.5370 & 2.1519 & 12.4741 & 12.2070 & 2.1881 \\
\hline & & 4 & 19.4217 & 20.6330 & -5.8707 & 17.8921 & 18.6300 & -3.9608 \\
\hline & \multirow{4}{*}{$\mathrm{CCCC}$} & 1 & 9.9436 & 9.6354 & 3.1986 & 8.9825 & 8.7011 & 3.2341 \\
\hline & & 2 & 19.1612 & 18.3050 & 4.6774 & 17.3531 & 16.5670 & 4.7450 \\
\hline & & 3 & 19.1612 & 18.3050 & 4.6774 & 17.3531 & 16.5670 & 4.7450 \\
\hline & & 4 & 26.8640 & 25.5060 & 5.3242 & 24.3601 & 23.1110 & 5.4048 \\
\hline
\end{tabular}


Table 3 Variation of the Frequency Parameter $\left(\bar{\omega}=\omega\left(a^{2} / h\right) \sqrt{\rho_{c} / E_{c}}\right)$ with the Volume Fraction Exponent $\varphi_{(1,2) \text { for plate } \mathrm{Al} / \mathrm{Al}_{2} \mathrm{O}_{3}(\mathrm{a} / \mathrm{h}=10)}$

\begin{tabular}{|c|c|c|c|c|c|c|c|c|}
\hline \multirow{2}{*}{$\begin{array}{c}\text { FGM } \\
\text { TYPE }\end{array}$} & \multirow[b]{2}{*}{ BCC } & \multirow[b]{2}{*}{ MODE } & \multicolumn{3}{|c|}{1} & \multicolumn{3}{|c|}{2} \\
\hline & & & Present & $\begin{array}{c}\text { Zhao et. } \\
\text { al. [8] }\end{array}$ & \%Error & Present & $\begin{array}{c}\text { Zhao et. } \\
\text { al. [8] }\end{array}$ & \% Error \\
\hline \multirow{8}{*}{ FGM1 } & \multirow{4}{*}{ SSSS } & 1 & 4.9476 & 4.8713 & 1.5663 & 4.7681 & 4.6977 & 1.4986 \\
\hline & & 2 & 11.8748 & 11.6330 & 2.0786 & 11.4140 & 11.1990 & 1.9198 \\
\hline & & 3 & 11.8759 & 11.6330 & 2.0880 & 11.4150 & 11.1990 & 1.9287 \\
\hline & & 4 & 17.0304 & 17.7480 & -4.0433 & 16.0931 & 17.0630 & -5.6842 \\
\hline & \multirow{4}{*}{$\mathrm{CCCC}$} & 1 & 8.5535 & 8.2925 & 3.1474 & 8.2117 & 7.9756 & 2.9603 \\
\hline & & 2 & 16.5110 & 15.7830 & 4.6126 & 15.8010 & 15.1470 & 4.3177 \\
\hline & & 3 & 16.5110 & 15.7830 & 4.6126 & 15.8010 & 15.1470 & 4.3177 \\
\hline & & 4 & 23.1665 & 22.0140 & 5.2353 & 22.1334 & 21.0990 & 4.9026 \\
\hline \multirow{8}{*}{ FGM2 } & \multirow{4}{*}{ SSSS } & 1 & 4.9485 & 4.8713 & 1.5848 & 4.7691 & 4.6977 & 1.5199 \\
\hline & & 2 & 11.8796 & 11.6330 & 2.1198 & 11.4193 & 11.1990 & 1.9671 \\
\hline & & 3 & 11.8806 & 11.6330 & 2.1284 & 11.4202 & 11.1990 & 1.9752 \\
\hline & & 4 & 17.0326 & 17.7480 & -4.0309 & 16.0955 & 17.0630 & -5.6702 \\
\hline & \multirow{4}{*}{ CCCC } & 1 & 8.5551 & 8.2925 & 3.1667 & 8.2134 & 7.9756 & 2.9816 \\
\hline & & 2 & 16.5173 & 15.7830 & 4.6525 & 15.8078 & 15.1470 & 4.3626 \\
\hline & & 3 & 16.5173 & 15.7830 & 4.6525 & 15.8078 & 15.1470 & 4.3626 \\
\hline & & 4 & 23.1787 & 22.0140 & 5.2907 & 22.1464 & 21.0990 & 4.9642 \\
\hline
\end{tabular}

Table 4: Variation of the Frequency Parameter $\left(\bar{\omega}=\omega\left(a^{2} / h\right) \sqrt{\rho_{c} / E_{c}}\right)$ with the Volume Fraction Exponent $\varphi_{(5,8,10) \text { for plate } \mathrm{Al} / \operatorname{AL2O3}(\mathrm{a} / \mathrm{h}=10)}$

\begin{tabular}{|c|c|c|c|c|c|c|c|c|c|c|c|}
\hline \multirow[b]{2}{*}{$\begin{array}{l}\text { FGM } \\
\text { TYPE }\end{array}$} & \multirow[b]{2}{*}{ BCC } & \multirow[b]{2}{*}{ MODE } & \multicolumn{3}{|c|}{5} & \multicolumn{3}{|c|}{8} & \multicolumn{3}{|c|}{10} \\
\hline & & & Present & $\begin{array}{c}\text { R Zhao } \\
\text { et. al. } \\
\text { [8] }\end{array}$ & \%Error & Present & $\begin{array}{c}\text { Zhao } \\
\text { et. al. } \\
{[8]}\end{array}$ & \%Error & Present & $\begin{array}{c}\text { Zhao } \\
\text { et. al. } \\
{[8]}\end{array}$ & \%Error \\
\hline \multirow{8}{*}{ FGM1 } & \multirow{4}{*}{ SSSS } & 1 & 4.6204 & 4.5549 & 1.4380 & 4.5396 & 4.4741 & 1.4640 & 4.4982 & 4.4323 & 1.4868 \\
\hline & & 2 & 11.0197 & 10.8280 & 1.7704 & 10.8255 & 10.6320 & 1.8200 & 10.7302 & 10.5330 & 1.8722 \\
\hline & & 3 & 11.0207 & 10.8280 & 1.7796 & 10.8264 & 10.6320 & 1.8284 & 10.7312 & 10.5330 & 1.8817 \\
\hline & & 4 & 15.0649 & 16.4620 & -8.4868 & 14.6983 & 16.1570 & -9.0283 & 14.5616 & 16.0080 & -9.0355 \\
\hline & \multirow{4}{*}{ CCCC } & 1 & 7.9088 & 7.6959 & 2.7664 & 7.7658 & 7.5521 & 2.8297 & 7.6975 & 7.4815 & 2.8871 \\
\hline & & 2 & 15.1475 & 14.5630 & 4.0136 & 14.8679 & 14.2820 & 4.1024 & 14.7418 & 14.1490 & 4.1897 \\
\hline & & 3 & 15.1475 & 14.5630 & 4.0136 & 14.8679 & 14.2820 & 4.1024 & 14.7418 & 14.1490 & 4.1897 \\
\hline & & 4 & 21.1681 & 20.2470 & 4.5493 & 20.7743 & 19.8490 & 4.6617 & 20.6016 & 19.6660 & 4.7574 \\
\hline \multirow{8}{*}{ FGM2 } & \multirow{4}{*}{ SSSS } & 1 & 4.6210 & 4.5549 & 1.4512 & 4.5400 & 4.4741 & 1.4729 & 4.4985 & 4.4323 & 1.4936 \\
\hline & & 2 & 11.0227 & 10.8280 & 1.7981 & 10.8273 & 10.6320 & 1.8369 & 10.7316 & 10.5330 & 1.8855 \\
\hline & & 3 & 11.0237 & 10.8280 & 1.8074 & 10.8282 & 10.6320 & 1.8454 & 10.7325 & 10.5330 & 1.8940 \\
\hline & & 4 & 15.0662 & 16.4620 & -8.4789 & 14.6990 & 16.1570 & -9.0240 & 14.5621 & 16.0080 & -9.0324 \\
\hline & \multirow{4}{*}{ CCCC } & 1 & 7.9098 & 7.6959 & 2.7794 & 7.7663 & 7.5521 & 2.8363 & $\begin{array}{l}7.6979 \\
\end{array}$ & 7.4815 & 2.8925 \\
\hline & & 2 & 15.1513 & 14.5630 & 4.0397 & 14.8702 & 14.2820 & 4.1185 & 14.7435 & 14.1490 & 4.2017 \\
\hline & & 3 & 15.1513 & 14.5630 & 4.0397 & 14.8702 & 14.2820 & 4.1185 & 14.7435 & 14.1490 & 4.2017 \\
\hline & & 4 & 21.1754 & 20.2470 & 4.5854 & 20.7787 & 19.8490 & 4.6839 & 20.6050 & 19.6660 & 4.7747 \\
\hline
\end{tabular}

\subsection{Numerical Examples}

\subsubsection{Spherical Shell}

Tables 5 and 6, show the non-dimensional frequency parameter variation with effect of the volume fraction index and Four parameter power law distribution $\mathrm{FGM} 1(\mathrm{i}=1, \mathrm{j}=0 / \mathrm{k} / \varphi)$ for constituent materials $\mathrm{Al} / \mathrm{Al}_{2} \mathrm{O}_{3}, \mathrm{Al} / \mathrm{ZrO}_{2}$ and the thickness ratio 
(a/ $\mathrm{h}=10)$, aspect ratio $(\mathrm{a} / \mathrm{b}=1)$, respectively. The volume fraction of the gradient was varied by changing the power law index. For the first four modes, results are computed. For the shell with different boundary condition SSSS and CCCC, the volume fraction exponent $\varphi$ increases, the frequencies in all four modes decreased. This is due to the fact that plate has a smaller ceramic component with a larger volume fraction exponent which reduces stiffness. A similar trend is observed for the CCCC boundary condition. The second power law distribution FGM2 $(i=1 / j=0 / k / \varphi)$ shows the power law index increases the response of non-dimensional frequency decreases. Clamped boundary conditions exhibit higher frequency responses compared to the simply supported conditions in both power law distribution.

Table 5: Variation of the Frequency Parameter $\left(\bar{\omega}=\omega\left(a^{2} / h\right) \sqrt{\rho_{c} / E_{c}}\right)$ with the Volume Fraction Exponent $\varphi$ for Spherical $\mathrm{Al}_{\mathbf{~} \mathrm{Al}_{2} \mathrm{O}_{3}(\mathrm{a} / \mathrm{h}=10)}$

\begin{tabular}{|c|c|c|c|c|c|c|c|c|c|}
\hline $\begin{array}{l}\text { FGM } \\
\text { TYPE }\end{array}$ & BCC & MODE & 0 & 0.5 & 1 & 2 & 5 & 8 & 10 \\
\hline \multirow{8}{*}{ FGM1 } & \multirow{4}{*}{ SSSS } & 1 & 6.0715 & 5.2017 & 4.7090 & 4.2715 & 3.9766 & 3.8698 & 3.8133 \\
\hline & & 2 & 13.9418 & 11.9243 & 10.7657 & 9.7333 & 9.0341 & $\begin{array}{l}8.7943 \\
\end{array}$ & 8.6736 \\
\hline & & 3 & 13.9431 & 11.9254 & 10.7666 & 9.7342 & 9.0349 & 8.7951 & 8.6744 \\
\hline & & 4 & 19.4217 & 17.3856 & 16.0843 & 14.5066 & 12.5325 & 11.7491 & 11.4433 \\
\hline & \multirow{4}{*}{ CCCC } & 1 & 10.3174 & 8.8690 & 8.0326 & 7.2616 & 6.6760 & 6.4676 & 6.3697 \\
\hline & & 2 & 19.2463 & 16.5991 & 15.0309 & 13.5444 & 12.3426 & 11.9260 & 11.7440 \\
\hline & & 3 & 19.2463 & 16.5991 & 15.0309 & 13.5444 & 12.3426 & 11.9260 & 11.7440 \\
\hline & & 4 & 26.9272 & 23.2664 & 21.0656 & 18.9390 & 17.1515 & 16.5428 & 16.2886 \\
\hline \multirow{8}{*}{ FGM2 } & \multirow{4}{*}{ SSSS } & 1 & 6.0715 & 5.1789 & 4.6783 & 4.2343 & 3.9388 & 3.8375 & 3.7844 \\
\hline & & 2 & 13.9418 & 11.8827 & 10.7135 & 9.6705 & 8.9652 & 8.7331 & 8.6181 \\
\hline & & 3 & 13.9431 & 11.8838 & 10.7145 & 9.6713 & 8.9660 & 8.7339 & 8.6189 \\
\hline & & 4 & 19.4217 & 17.4441 & 16.1715 & 14.6112 & 12.6210 & 11.8183 & 11.5032 \\
\hline & \multirow{4}{*}{ CCCC } & 1 & 10.3174 & 8.8495 & 8.0070 & 7.2297 & 6.6425 & 6.4391 & 6.3443 \\
\hline & & 2 & 19.2463 & 16.5453 & 14.9641 & 13.4641 & 12.2544 & 11.8476 & 11.6729 \\
\hline & & 3 & 19.2463 & 16.5453 & 14.9641 & 13.4641 & 12.2544 & 11.8476 & 11.6729 \\
\hline & & 4 & 26.9272 & 23.2125 & 21.0067 & 18.8675 & 17.0609 & 16.4587 & 09 \\
\hline
\end{tabular}

Table 6: Variation of the Frequency Parameter $\left(\bar{\omega}=\omega\left(a^{2} / h\right) \sqrt{\rho_{c} / E_{c}}\right)$ with the Volume Fraction Exponent $\varphi$ for Spherical $\mathrm{Al} / \mathrm{ZrO}_{2}$ FG shell $(\mathrm{a} / \mathrm{h}=10)$

\begin{tabular}{|c|c|c|c|c|c|c|c|c|c|}
\hline $\begin{array}{l}\text { FGM } \\
\text { TYPE }\end{array}$ & BCC & MODE & 0 & 0.5 & 1 & 2 & 5 & 8 & 10 \\
\hline \multirow{8}{*}{ FGM1 } & \multirow{4}{*}{ SSSS } & 1 & 5.8327 & 4.9677 & 4.4833 & 4.0657 & 3.8111 & 3.7221 & 3.6722 \\
\hline & & 2 & 13.8144 & 11.7932 & 10.6368 & 9.6138 & 8.9366 & 8.7077 & 8.5914 \\
\hline & & 3 & 13.9061 & 11.8657 & 10.6995 & 9.6649 & 8.9756 & 8.7451 & 8.6290 \\
\hline & & 4 & 19.4217 & 17.3856 & 16.0843 & 14.5066 & 12.5325 & 11.7491 & 11.4433 \\
\hline & \multirow{4}{*}{$\mathrm{CCCC}$} & 1 & 10.0947 & 8.6529 & 7.8247 & 7.0710 & 6.5199 & 6.3273 & 6.2356 \\
\hline & & 2 & 19.1529 & 16.4993 & 14.9311 & 13.4503 & 12.2648 & 11.8571 & 11.6790 \\
\hline & & 3 & 19.2417 & 16.5583 & 14.9768 & 13.4837 & 12.2907 & 11.8848 & 11.7083 \\
\hline & & 4 & 26.8790 & 23.1927 & 20.9839 & 18.8556 & 17.0801 & 16.4818 & 16.2326 \\
\hline \multirow{4}{*}{ FGM2 } & \multirow{4}{*}{ SSSS } & 1 & 5.8327 & 4.9580 & 4.4708 & 4.0506 & 3.7947 & 3.7075 & 3.6591 \\
\hline & & 2 & 13.8144 & 11.7688 & 10.6084 & 9.5791 & 8.8943 & 8.6688 & 8.5557 \\
\hline & & 3 & 13.9061 & 11.8635 & 10.7027 & 9.6691 & 8.9709 & 8.7370 & 8.6202 \\
\hline & & 4 & 19.4217 & 17.4279 & 16.1457 & 14.5784 & 12.5902 & 11.7929 & 11.4807 \\
\hline
\end{tabular}




\begin{tabular}{|l|c|c|c|c|c|c|c|c|c|}
\hline & 1 & 10.0947 & 8.6456 & 7.8164 & 7.0605 & 6.5067 & 6.3153 & 6.2247 \\
\cline { 2 - 10 } & 2 & 19.1529 & 16.4646 & 14.8911 & 13.4017 & 12.2058 & 11.8029 & 11.6292 \\
\cline { 2 - 10 } CCCC & 3 & 19.2417 & 16.5592 & 14.9867 & 13.4954 & 12.2892 & 11.8782 & 11.7005 \\
\cline { 2 - 9 } & 4 & 26.8790 & 23.1854 & 20.9903 & 18.8616 & 17.0608 & 16.4562 & 16.2066 \\
\hline
\end{tabular}

\subsubsection{Cylindrical Shell}

Tables 7 demonstrates the variation of frequency parameter with the volume fraction exponent for the $\mathrm{Al} / \mathrm{Al}_{2} \mathrm{O}_{3}$, and the geometric parameter thickness ratio $(\mathrm{a} / \mathrm{h}=10)$ and aspect ratio $(\mathrm{a} / \mathrm{b}=1)$ of the FGM1 and FGM2 distributions, respectively. Table 8 shows changes in non-dimensional frequencies with the power law index. A similar trend follows for both FGM1 and FGM2 distributions of the power law. Combining aluminum with alumina (Al / Al2O3) reveals a higher frequency response compared to aluminum and zirconia $(\mathrm{Al} / \mathrm{ZrO} 2$.) of the cylindrical shell panels. The gradients of the material properties (E, $\mathrm{P}$, and $\mathrm{U}$ ) playssignificant role in approximating the shell structure's response.

Table 7: Variation of the Frequency Parameter $\left(\bar{\omega}=\omega\left(a^{2} / h\right) \sqrt{\rho_{c} / E_{c}}\right)$ with the Volume Fraction Exponent $\varphi$ for Cylindrical $\mathrm{Al} / \mathrm{Al}_{2} \mathrm{O}_{3}$ FG Shell $(\mathrm{a} / \mathrm{h}=10)$

\begin{tabular}{|c|c|c|c|c|c|c|c|c|c|}
\hline $\begin{array}{l}\text { FGM } \\
\text { TYPE }\end{array}$ & BCC & MODE & 0 & 0.5 & 1 & 2 & 5 & 8 & 10 \\
\hline \multirow{8}{*}{ FGM1 } & \multirow{4}{*}{ SSSS } & 1 & 6.0715 & 5.4887 & 5.2332 & 5.0344 & 4.8586 & 4.7679 & 4.7232 \\
\hline & & 2 & 13.9418 & 12.5987 & 12.0049 & 11.5388 & 11.1279 & 10.9250 & 10.8263 \\
\hline & & 3 & 13.9431 & 12.5999 & 12.0060 & 11.5398 & 11.1289 & 10.9260 & 10.8273 \\
\hline & & 4 & 19.4217 & 17.8729 & 17.0067 & 16.0680 & 15.0457 & 14.6836 & 14.5489 \\
\hline & \multirow{4}{*}{$\mathrm{CCCC}$} & 1 & 10.3174 & 9.3409 & 8.8989 & 8.5354 & 8.2018 & 8.0475 & 7.9753 \\
\hline & & 2 & 19.2463 & 17.4564 & 16.6231 & 15.9103 & 15.2403 & 14.9510 & 14.8208 \\
\hline & & 3 & 19.2463 & 17.4564 & 16.6231 & 15.9103 & 15.2403 & 14.9510 & 14.8208 \\
\hline & & 4 & 26.9272 & 24.4452 & 23.2671 & 22.2337 & 21.2520 & 20.8474 & 20.6701 \\
\hline \multirow{8}{*}{ FGM2 } & \multirow{4}{*}{ SSSS } & 1 & 6.0715 & 5.4732 & 5.2122 & 5.0109 & 4.8399 & 4.7539 & 4.7113 \\
\hline & & 2 & 13.9418 & 12.5661 & 11.9614 & 11.4902 & 11.0889 & 10.8954 & 10.8012 \\
\hline & & 3 & 13.9431 & 12.5673 & 11.9625 & 11.4912 & 11.0899 & 10.8964 & 10.8022 \\
\hline & & 4 & 19.4217 & 17.9053 & 17.0504 & 16.1152 & 15.0818 & 14.7111 & 14.5725 \\
\hline & \multirow{4}{*}{$\mathrm{CCCC}$} & 1 & 10.3174 & 9.3264 & 8.8791 & 8.5131 & 8.1843 & 8.0346 & 7.9646 \\
\hline & & 2 & 19.2463 & 17.4139 & 16.5665 & 15.8474 & 15.1898 & 14.9126 & 14.7880 \\
\hline & & 3 & 19.2463 & 17.4139 & 16.5665 & 15.8474 & 15.1898 & 14.9126 & 14.7880 \\
\hline & & 4 & 26.9272 & 24.3944 & 23.2005 & 22.1598 & 21.1921 & 20.8014 & 20.6308 \\
\hline
\end{tabular}

Table 8: Variation of the Frequency Parameter $\left(\bar{\omega}=\omega\left(a^{2} / h\right) \sqrt{\rho_{c} / E_{c}}\right)$ with the Volume Fraction Exponent $\varphi$ for Cylindrical $\mathrm{Al} / \mathrm{ZrO}_{2}$ FG Shell $(\mathrm{a} / \mathrm{h}=10)$

\begin{tabular}{|c|c|c|c|c|c|c|c|c|c|}
\hline $\begin{array}{c}\text { FGM } \\
\text { TYPE }\end{array}$ & BCC & MODE & $\mathbf{0}$ & $\mathbf{0 . 5}$ & $\mathbf{1}$ & $\mathbf{2}$ & $\mathbf{5}$ & $\mathbf{8}$ & $\mathbf{1 0}$ \\
\hline \multirow{3}{*}{ FGM1 } & \multirow{2}{*}{ SSSS } & 1 & 5.8327 & 5.2592 & 5.0131 & 4.8293 & 4.6739 & 4.5902 & 4.5477 \\
\cline { 3 - 10 } & & 2 & 13.8144 & 12.4727 & 11.8828 & 11.4241 & 11.0251 & 10.8269 & 10.7298 \\
\cline { 2 - 9 } & & 13.9061 & 12.5508 & 11.9538 & 11.4884 & 11.0857 & 10.8878 & 10.7912 \\
\hline
\end{tabular}




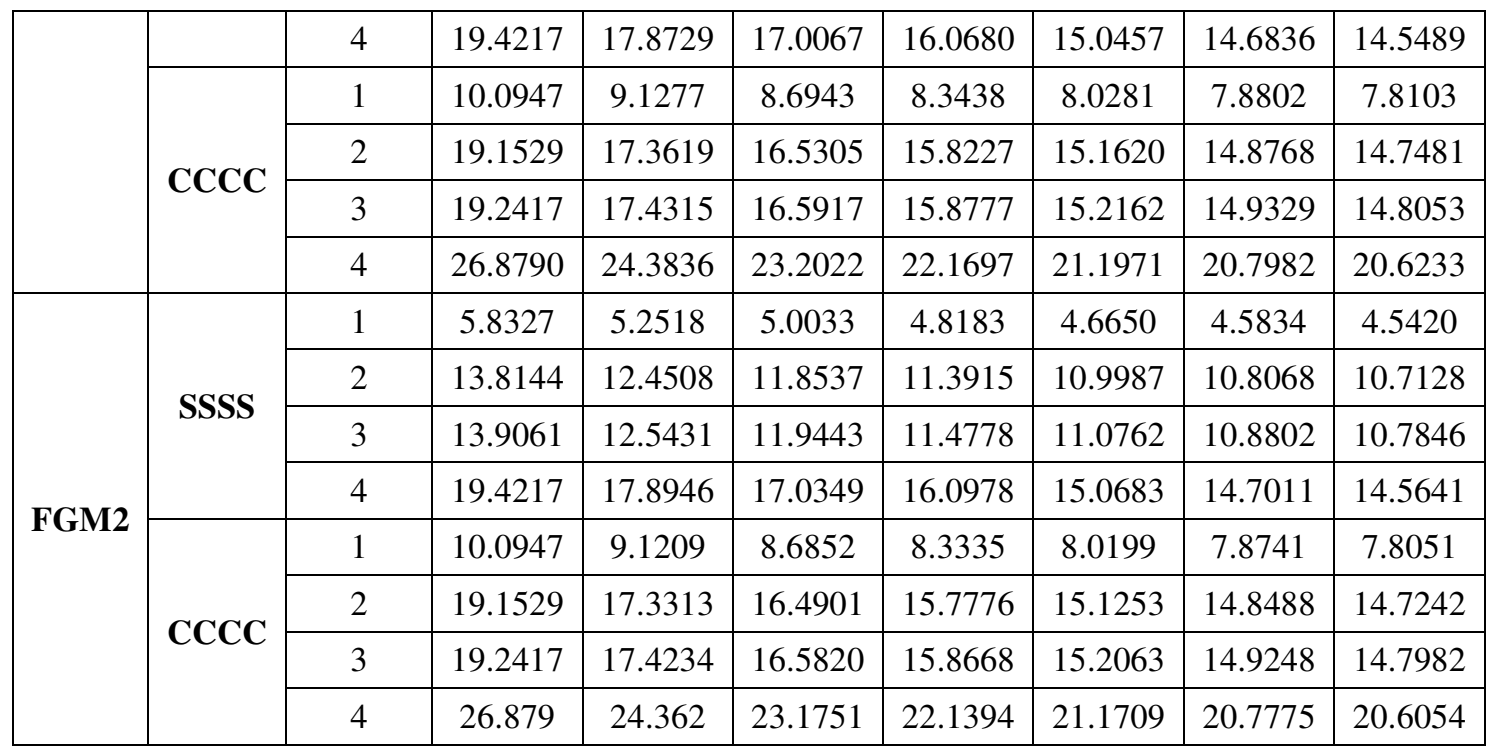

\subsubsection{HyperboloidShell}

Tables 9 and 10, show the variety of the non-dimensional frequency parameter with the volume fraction index for FGM constituents $\mathrm{Al} / \mathrm{Al} 2 \mathrm{O} 3, \mathrm{Al} / \mathrm{ZrO} 2$ hyperboloid shell, and the thickness proportion $(\mathrm{a} / \mathrm{h}=10),(\mathrm{a} / \mathrm{b}=1)$, respectively.The first four modes are considered for the study. It is additionally intriguing to take note that the frequency responses show a decreasing pattern from ceramic rich to metal-rich FG structures. It is because; the ceramic materials are higher firmness rather than the metal surface. Also, Supporting conditions impact on the variation of non-dimensional frequencies. The second power-law circulation $\operatorname{FGM} 2(\mathrm{i}=1 / \mathrm{j}=0 / \mathrm{k} / \varphi)$ shows the power-law exponent increases the reaction of nondimensional frequencies diminishes. FGM1 and FGM2 input parameters given similar qualities. It is clear that FGM1 power-law dissemination display higher frequency responses contrasted with the FGM2 responses.

Table 9: Variation of the Frequency Parameter $\left(\bar{\omega}=\omega\left(a^{2} / h\right) \sqrt{\rho_{c} / E_{c}}\right)$ with the Volume Fraction Exponent $\varphi$ for Hyper $\mathrm{Al} / \mathrm{Al}_{2} \mathrm{O}_{3}$ FG $\operatorname{shell}(\mathrm{a} / \mathrm{h}=10)$

\begin{tabular}{|c|c|c|c|c|c|c|c|c|c|}
\hline $\begin{array}{l}\text { FGM } \\
\text { TYPE }\end{array}$ & BCC & MODE & 0 & 0.5 & 1 & 2 & 5 & 8 & 10 \\
\hline \multirow{8}{*}{ FGM1 } & \multirow{4}{*}{ SSSS } & 1 & 5.7189 & 4.8556 & 4.3749 & 3.9653 & 3.7263 & 3.6451 & 3.5984 \\
\hline & & 2 & 13.8425 & 11.7850 & 10.6144 & 9.5796 & $\begin{array}{l}8.8999 \\
\end{array}$ & 8.6782 & 8.5666 \\
\hline & & 3 & 13.8437 & 11.8120 & 10.6511 & 9.6232 & 8.9410 & 8.7119 & 8.5960 \\
\hline & & 4 & 19.4217 & 17.3856 & 16.0843 & 14.5066 & 12.5326 & 11.7491 & 11.4433 \\
\hline & \multirow{4}{*}{$\mathrm{CCCC}$} & 1 & 10.1690 & 8.7157 & 7.8811 & 7.1190 & 6.5573 & 6.3622 & 6.2698 \\
\hline & & 2 & 19.2201 & 16.5072 & 14.9159 & 13.4173 & 12.2298 & 11.8324 & 11.6605 \\
\hline & & 3 & 19.2201 & 16.5498 & 14.9739 & 13.4851 & 12.2925 & 11.8841 & 11.7062 \\
\hline & & 4 & 26.8607 & 23.1502 & 20.9334 & 18.8008 & 17.0301 & 16.4386 & 16.1930 \\
\hline \multirow{8}{*}{ FGM2 } & \multirow{4}{*}{ SSSS } & 1 & 5.7189 & 4.8583 & 4.3799 & 3.9713 & 3.7306 & 3.6478 & 3.6006 \\
\hline & & 2 & 13.8425 & 11.8045 & 10.6464 & 9.6166 & 8.9252 & 8.6950 & 8.5799 \\
\hline & & 3 & 13.8437 & 11.8218 & 10.6724 & 9.6504 & 8.9590 & $\begin{array}{l}8.7229 \\
\end{array}$ & 8.6042 \\
\hline & & 4 & 19.4217 & 17.4092 & 16.1176 & 14.5435 & 12.5577 & 11.7659 & 11.4567 \\
\hline & \multirow{4}{*}{$\mathrm{CCCC}$} & 1 & 10.1690 & 8.7207 & 7.8903 & 7.1301 & 6.5646 & 6.3668 & 6.2734 \\
\hline & & 2 & 19.2201 & 16.5342 & 14.9605 & 13.4681 & 12.2626 & 11.8539 & 11.6776 \\
\hline & & 3 & 19.2201 & 16.5627 & 15.0022 & 13.5206 & 12.3147 & 11.8975 & 11.7162 \\
\hline & & 4 & 26.8607 & 23.1892 & 21.0045 & 18.8839 & 17.0815 & 16.4712 & 16.2184 \\
\hline
\end{tabular}


Table 10: Variation of the Frequency Parameter $\left(\bar{\omega}=\omega\left(a^{2} / h\right) \sqrt{\rho_{c} / E_{c}}\right)$ with the Volume Fraction Exponent $\varphi$ for Hyper $\mathrm{Al} / \mathrm{ZrO}_{2}$ FG shell $(\mathrm{a} / \mathrm{h}=10)$

\begin{tabular}{|c|c|c|c|c|c|c|c|c|c|}
\hline $\begin{array}{l}\text { FGM } \\
\text { TYPE }\end{array}$ & BCC & MODE & 0 & 0.5 & 1 & 2 & 5 & 8 & 10 \\
\hline \multirow{8}{*}{ FGM1 } & \multirow{4}{*}{ SSSS } & 1 & 5.7189 & 5.1489 & 4.9065 & 4.7285 & 4.5818 & 4.5017 & 4.4606 \\
\hline & & 2 & 13.8425 & 12.4785 & 11.8795 & 11.4154 & 11.0206 & 10.8281 & 10.7338 \\
\hline & & 3 & 13.8437 & 12.4950 & 11.9017 & 11.4399 & 11.0397 & 10.8423 & 10.7458 \\
\hline & & 4 & 19.4217 & 17.8729 & 17.0067 & 16.0680 & 15.0457 & 14.6836 & 14.5489 \\
\hline & \multirow{4}{*}{ CCCC } & 1 & 10.1690 & 9.1933 & 8.7550 & 8.3991 & 8.0794 & 7.9311 & 7.8612 \\
\hline & & 2 & 19.2201 & 17.3930 & 16.5476 & 15.8317 & 15.1771 & 14.8996 & 14.7747 \\
\hline & & 3 & 19.2201 & 17.4180 & 16.5814 & 15.8690 & 15.2060 & 14.9211 & 14.7928 \\
\hline & & 4 & 26.8607 & 24.3515 & 23.1654 & 22.1313 & 21.1640 & 20.7697 & 20.5971 \\
\hline \multirow{8}{*}{ FGM2 } & \multirow{4}{*}{ SSSS } & 1 & 5.7189 & 5.1494 & 4.9074 & 4.7295 & 4.5824 & 4.5020 & 4.4608 \\
\hline & & 2 & 13.8425 & 12.4837 & 11.8869 & 11.4231 & 11.0257 & 10.8316 & 10.7368 \\
\hline & & 3 & 13.8437 & 12.4954 & 11.9038 & 11.4425 & 11.0406 & 10.8423 & 10.7455 \\
\hline & & 4 & 19.4217 & 17.8814 & 17.0170 & 16.0782 & 15.0529 & 14.6891 & 14.5538 \\
\hline & \multirow{4}{*}{$\mathrm{CCCC}$} & 1 & 10.1690 & 9.1943 & 8.7566 & 8.4009 & 8.0804 & 7.9317 & 7.8617 \\
\hline & & 2 & 19.2201 & 17.3992 & 16.5569 & 15.8413 & 15.1829 & 14.9034 & 14.7777 \\
\hline & & 3 & 19.2201 & 17.4194 & 16.5848 & 15.8729 & 15.2078 & 14.9219 & 14.7933 \\
\hline & & 4 & 26.8607 & 24.3588 & 23.1775 & 22.1442 & 21.1714 & 20.7742 & 20.6004 \\
\hline
\end{tabular}

\subsubsection{Elliptical Shell}

Tables 11 and 12 show the variation of the non-dimensional frequency parameter with the volume fraction exponent for the $\mathrm{Al} / \mathrm{Al}_{2} \mathrm{O}_{3}, \mathrm{Al} / \mathrm{ZrO}_{2}$ elliptical shell, and the thickness ratio $(\mathrm{a} / \mathrm{h}=10)$ and $(\mathrm{a} / \mathrm{b}=1)$, respectively. For the shell with SSSS, CCCC boundary condition, the volume fraction exponent $\varphi$ decreases when $\varphi=0$ (ceramic)and $(\varphi=\infty)$ for metal. The second power law distribution $\mathrm{FGM} 2(\mathrm{i}=1 / \mathrm{j}=0 / \mathrm{k} / \varphi)$ shows the power law index increases the response of non-dimensional frequency decreases. Clamped boundary conditions exhibit higher frequency responses compared to the simply supported conditions.

Table 11: Variation of the Frequency Parameter $\left(\bar{\omega}=\omega\left(a^{2} / h\right) \sqrt{\rho_{c} / E_{c}}\right)$ with the Volume Fraction Exponent $\varphi$ for Elliptical $\mathrm{Al} / \mathrm{Al}_{2} \mathrm{O}_{3}$ FG shell $(\mathrm{a} / \mathrm{h}=10)$

\begin{tabular}{|c|c|c|c|c|c|c|c|c|c|}
\hline $\begin{array}{l}\text { FGM } \\
\text { TYPE }\end{array}$ & BCC & MODE & 0 & 0.5 & 1 & 2 & 5 & 8 & 10 \\
\hline \multirow{8}{*}{ FGM1 } & \multirow{4}{*}{ SSSS } & 1 & 5.9374 & 5.0704 & 4.5825 & 4.1563 & 3.8844 & 3.7876 & 3.7348 \\
\hline & & 2 & 13.8592 & 11.8406 & 10.6838 & 9.6579 & 8.9730 & 8.7400 & 8.6220 \\
\hline & & 3 & 13.9274 & 11.8978 & 10.7350 & 9.7013 & 9.0071 & 8.7720 & 8.6535 \\
\hline & & 4 & 19.4217 & 17.3856 & 16.0843 & 14.5066 & 12.5325 & 11.7491 & 11.4433 \\
\hline & \multirow{4}{*}{ СССС } & 1 & 10.1696 & 8.7267 & 7.8963 & 7.1371 & 6.5742 & 6.3760 & 6.2819 \\
\hline & & 2 & 19.1796 & 16.5304 & 14.9632 & 13.4813 & 12.2905 & 11.8796 & 11.7000 \\
\hline & & 3 & 19.2461 & 16.5801 & 15.0048 & 13.5148 & 12.3179 & 11.9069 & 11.7277 \\
\hline & & 4 & 26.8994 & 23.2257 & 21.0209 & 18.8938 & 17.1132 & 16.5101 & 16.2586 \\
\hline \multirow{3}{*}{ FGM2 } & \multirow{3}{*}{ SSSS } & 1 & 5.9374 & 5.0543 & 4.5610 & 4.1302 & 3.8573 & 3.7642 & 3.7139 \\
\hline & & 2 & 13.8592 & 11.8076 & 10.6436 & 9.6092 & 8.9175 & 8.6900 & 8.5765 \\
\hline & & 3 & 13.9274 & 11.8759 & 10.7105 & 9.6719 & 8.9702 & 8.7373 & 8.6214 \\
\hline
\end{tabular}




\begin{tabular}{|c|c|c|c|c|c|c|c|c|c|}
\hline & 4 & 19.4217 & 17.4372 & 16.1597 & 14.5958 & 12.6064 & 11.8064 & 11.4927 \\
\cline { 2 - 9 } & & 1 & 10.1696 & 8.7134 & 7.8793 & 7.1159 & 6.5508 & 6.3557 & 6.2638 \\
\cline { 2 - 9 } & 2 & 19.1796 & 16.4862 & 14.9099 & 13.4169 & 12.2170 & 11.8134 & 11.6396 \\
\cline { 2 - 9 } & 3 & 19.2461 & 16.5535 & 14.9763 & 13.4804 & 12.2729 & 11.8642 & 11.6881 \\
\cline { 2 - 9 } & 4 & 26.8994 & 23.1951 & 20.9946 & 18.8610 & 17.0582 & 16.4553 & 16.2067 \\
\hline
\end{tabular}

Table 12: Variation of the Frequency Parameter $\left(\bar{\omega}=\omega\left(a^{2} / h\right) \sqrt{\rho_{c} / E_{c}}\right)$ with the Volume Fraction Exponent $\varphi$ for Elliptical Al/ $\mathrm{ZrO}_{2}$ FG Shell (a/h=10)

\begin{tabular}{|c|c|c|c|c|c|c|c|c|c|}
\hline $\begin{array}{l}\text { FGM } \\
\text { TYPE }\end{array}$ & BCC & MODE & 0 & 0.5 & 1 & 2 & 5 & 8 & 10 \\
\hline \multirow{8}{*}{ FGM1 } & \multirow{4}{*}{ SSSS } & 1 & 5.9374 & 5.3599 & 5.1099 & 4.9196 & 4.7554 & 4.6685 & 4.6250 \\
\hline & & 2 & 13.8592 & 12.5178 & 11.9267 & 11.4657 & 11.0623 & 10.8622 & 10.7645 \\
\hline & & 3 & 13.9274 & 12.5779 & 11.9823 & 11.5165 & 11.1097 & 10.9093 & 10.8116 \\
\hline & & 4 & 19.4217 & 17.8729 & 17.0067 & 16.0680 & 15.0457 & 14.6836 & 14.5489 \\
\hline & \multirow{4}{*}{ СCCC } & 1 & 10.1696 & 9.2001 & 8.7641 & 8.4092 & 8.0873 & 7.9370 & 7.8663 \\
\hline & & 2 & 19.1796 & 17.3904 & 16.5589 & 15.8498 & 15.1859 & 14.8991 & 14.7699 \\
\hline & & 3 & 19.2461 & 17.4457 & 16.6091 & 15.8957 & 15.2302 & 14.9439 & 14.8150 \\
\hline & & 4 & 26.8994 & 24.4107 & 23.2312 & 22.1986 & 21.2219 & 20.8202 & 20.6442 \\
\hline \multirow{8}{*}{ FGM2 } & \multirow{4}{*}{ SSSS } & 1 & 5.9374 & 5.3485 & 5.0945 & 4.9024 & 4.7416 & 4.6582 & 4.6163 \\
\hline & & 2 & 13.8592 & 12.4906 & 11.8905 & 11.4251 & 11.0296 & 10.8374 & 10.7435 \\
\hline & & 3 & 13.9274 & 12.5577 & 11.9557 & 11.4868 & 11.0854 & 10.8906 & 10.7957 \\
\hline & & 4 & 19.4217 & 17.9012 & 17.0439 & 16.1076 & 15.0761 & 14.7071 & 14.5693 \\
\hline & \multirow{4}{*}{ ССCC } & 1 & 10.1696 & 9.1895 & 8.7496 & 8.3930 & 8.0745 & 7.9276 & 7.8583 \\
\hline & & 2 & 19.1796 & 17.3539 & 16.5105 & 15.7959 & 15.1424 & 14.8659 & 14.7416 \\
\hline & & 3 & 19.2461 & 17.4203 & 16.5758 & 15.8587 & 15.1999 & 14.9206 & 14.7950 \\
\hline & & 4 & 26.8994 & 24.3746 & 23.1843 & 22.1464 & 21.1788 & 20.7869 & 20.6155 \\
\hline
\end{tabular}

\section{CONCLUSIONS}

Linear vibration analysis of the static evaluation of the rectangular FG double curved panel has been investigated using MATLAB16a programming configuration.Governing equation structures are derived by the process of the variation principle. The higher order mathematical model uses TSDT kinematics and a combination of the finite element form. Finally, the relevance of the current model is established by solving various types of numerical case studies for different materials, power law index, boundary conditions, and geometric configurations.

- The frequency parameters of the clamped boundary conditions produced the highest frequency response relative to other general boundary conditions.

- The two power-law dispersions on the curved shell panels demonstrate the vibration characteristics of the structure, and the vibration response of the non-dimensional frequencies is distinct.

- As the power-law index is increased, the frequency responses are following the trend in the reverse directions for all the FG shell structures.

- Shell with cylindrical structure has a maximum frequency and minimum is found for an FG plate

- Ceramic to metal-rich FG surfaces structure has a higher frequency parameter compared to metal-rich to ceramic 
FG structure. The combination of material distribution parameters $\mathrm{i} / \mathrm{j} / \mathrm{k} /$ and $\varphi$ determines to characterize the volume fraction profiles. Nevertheless, with the rise in the power-law index, the frequency reactions fall apart.

\section{REFERENCES}

1. B. N. Pandya and T. Kant, "Finite element analysis of laminated composite plates using a higher-order displacement model," Compos. Sci. Technol., vol. 32, no. 2, pp. 137-155, 1988.

2. J. N. Reddy, “Analysis of functionally graded plates,” Int. J. Numer. Methods Eng., vol. 47, no. 1-3, pp. 663-684, 2000.

3. J. N. Reddy and Z. Q. Cheng, "Frequency correspondence between membranes and functionally graded spherical shallow shells of polygonal planform," Int. J. Mech. Sci., vol. 44, no. 5, pp. 967-985, 2002.

4. R. C. Batra and S. Aimmanee, "Vibrations of thick isotropic plates with higher order shear and normal deformable Plate theories," Comput. Struct., vol. 83, no. 12-13, pp. 934-955, 2005.

5. Y. W. Kim, “Temperature dependent vibration analysis of functionally graded rectangular plates, ”. Sound Vib., vol. 284, no. 3-5, pp. 531-549, 2005.

6. S. Kandasamy and A. V. Singh, "Free vibration analysis of skewed open circular cylindrical shells," J. Sound Vib., vol. 290, no. 3-5, pp. 1100-1118, 2006.

7. F. Tornabene and E. Viola, "2-D solution for free vibrations of parabolic shells using generalized differential quadrature method,” Eur. J. Mech. A/Solids, vol. 27, no. 6, pp. 1001-1025, 2008.

8. X. Zhao, Y. Y. Lee, and K. M. Liew, "Free vibration analysis of functionally graded plates using the element-free kp-Ritz method," J. Sound Vib., vol. 319, no. 3-5, pp. 918-939, 2009.

9. F. Tornabene, "Free vibration analysis of functionally graded conical, cylindrical shell and annular plate structures with a four-parameter power-law distribution," Comput. Methods Appl. Mech. Eng., vol. 198, no. 37-40, pp. 2911-2935, 2009.

10. S. Hosseini-Hashemi, H. RokniDamavandiTaher, H. Akhavan, and M. Omidi, "Free vibration of functionally graded rectangular plates using first-order shear deformation plate theory,” Appl. Math. Model., vol. 34, no. 5, pp. 1276-1291, 2010.

11. M. Talha and B. N. Singh, "Static response and free vibration analysis of FGM plates using higher order shear deformation theory,” Appl. Math. Model., vol. 34, no. 12, pp. 3991-4011, 2010.

12. K. M. Liew, X. Zhao, and A. J. M. Ferreira, "A review of meshless methods for laminated and functionally graded plates and shells, Compos. Struct., vol. 93, no. 8, pp. 2031-2041, 2011.

13. M. Ruan, Z. M. Wang, and Y. Wang, "Dynamic stability of functionally graded materials skew plates subjected to uniformly distributed tangential follower forces," JVC/Journal Vib. Control, vol. 18, no. 7, pp. 913-923, 2012.

14. F. Tornabene, A. Liverani, and G. Caligiana, "Laminated composite rectangular and annular plates: A GDQ solution for static analysis with a posteriori shear and normal stress recovery,” Compos. Part B Eng., vol. 43, no. 4, pp. 1847-1872, 2012.

15. D. K. Jha, T. Kant, and R. K. Singh, “A critical review of recent research on functionally graded plates,” Compos. Struct., vol. 96, pp. 833-849, 2013.

16. M. Shariyat and S. H. Hosseini, "Eccentric impact analysis of pre-stressed composite sandwich plates with viscoelastic cores: A novel global-local theory and a refined contact law,” Compos. Struct., vol. 117, no. 1, pp. 333-345, 2014.

17. GulshanTaj M. N.A., A. Chakrabarti, and V. Prakash, "Vibration Characteristics of Functionally Graded Material Skew Plate in Thermal Environment,” Int. J. Mech. ,Aerospace,Industrial, Mechatronics Manuf. Eng., vol. 8, no. 1, pp. 142-153, 2014. 
18. X. Xie, H. Zheng, and G. Jin, "Free vibration of four-parameter functionally graded spherical and parabolic shells of revolution with arbitrary boundary conditions," Compos. Part B Eng., vol. 77, pp. 59-73, 2015.

19. V. R. Kar and S. K. Panda, "Free vibration responses of temperature dependent functionally graded curved panels under thermal environment,” Lat. Am. J. Solids Struct., vol. 12, no. 11, pp. 2006-2024, 2015.

20. C. Zhang, G. Jin, X. Ma, and T. Ye, "Vibration analysis of circular cylindrical double-shell structures under general coupling and end boundary conditions," Appl. Acoust., vol. 110, pp. 176-193, 2016.

21. Q. Wang, D. Shi, Q. Liang, and F. Pang, "Free vibration of four-parameter functionally graded moderately thick doublycurved panels and shells of revolution with general boundary conditions,” Appl. Math. Model., vol. 42, pp. 705-734, 2017.

22. F. ZareJouneghani, R. Dimitri, M. Bacciocchi, and F. Tornabene, "Free Vibration Analysis of Functionally Graded Porous Doubly-Curved Shells Based on the First-Order Shear Deformation Theory,” Appl. Sci., vol. 7, no. 12, 2017.

23. A. S. Rezaei, A. R. Saidi, M. Abrishamdari, and M. H. P. Mohammadi, "Natural frequencies of functionally graded plates with porosities via a simple four variable plate theory: An analytical approach,” Thin-Walled Struct., vol. 120, no. May, pp. 366$377,2017$.

24. J. S. Moita, A. L. Araújo, V. F. Correia, C. M. MotaSoares, and J. Herskovits, "Active-passive damping in functionally graded sandwich plate/shell structures,” Compos. Struct., vol. 202, no. January, pp. 324-332, 2018.

25. S. Chakraverty and K. K. Pradhan, "Flexural vibration of functionally graded thin skew plates resting on elastic foundations," Int. J. Dyn. Control, vol. 6, no. 1, pp. 97-121, 2018.

26. C. M. Badiganti and R. Koona, "Harmonic frequency analysis of skewed functionally graded flat and spherical shallow shells,” Results Phys., vol. 10, no. June, pp. 987-992, 2018.

27. P. V. Katariya, C. K. Hirwani, and S. K. Panda, "Geometrically nonlinear deflection and stress analysis of skew sandwich shell panel using higher-order theory," Eng. Comput., vol. 35, no. 2, pp. 467-485, 2019.

28. P. V. Katariya and S. K. Panda, "Frequency and Deflection Responses of Shear Deformable Skew Sandwich Curved Shell Panel: A Finite Element Approach,” Arab. J. Sci. Eng., vol. 44, no. 2, pp. 1631-1648, 2019.

29. M. Rezaiee-Pajand, D. Pourhekmat, and E. Arabi, “Thermo-mechanical stability analysis of functionally graded shells,” Eng. Struct., vol. 178, no. September 2018, pp. 1-11, 2019. 